\title{
An Investigation into Worldwide Interoperability for Microwave Access (WIMAX) Across the Local Loop
}

\author{
Mohammad Arifin Rahman Khan ${ }^{1}$, Md. Sadiq Iqbal ${ }^{2}$ \\ Lecturer, CSE Department, Bangladesh University, Dhaka, Bangladesh ${ }^{1}$ \\ Assistant Professor, CSE Department, Bangladesh University, Dhaka, Bangladesh ${ }^{2}$
}

\begin{abstract}
The term "wireless" is used to describe communication in which electromagnetic waves or Radio Frequency carries a signal over a communication path. It allows the transfer of information without the use of wires. An access network in the local loop or the last mile is based on the principle of having continuous connectivity between the telephone exchange and the end customer. Over the local loop different technologies can be used to provide a telecommunications based service such as, microwave, radio, fibre or even electricity power cables. In this report, we will concentrate on the new and developing technology called WiMAX - Worldwide Interoperability for microwave access.
\end{abstract}

Keywords: Wireless Metropolitan Area Network (WMAN), Time Division Multiplexing (TDM), Voice-Over-Internet Protocol (VOIP), Quality of Service, Orthogonal Frequency Division Multiplexing (OFDM)

\section{INTRODUCTION}

Over the years, wireless technology has gained popularity in a number of sectors: including healthcare, education, retail, manufacturing and warehousing. With the use of hand-held devices and notebook computers to transmit real-time information to centralized hosts for processing, these industries have profited a great deal. Users are able to access shared information without having to contend with network cables, network managers are able to set up rapidly adapt networks without installing or moving wires. Wireless networks are nothing new, they have been around for many years, the most common of these networks to date is wireless LAN's and the Wi-Fi hotspot these two technologies uses a wireless broadband type service and adhere to the 802.11 (a ,b or g) standards [1].

Moreover, Broadband access not only provides fast Web surfing and quick file downloads, but it additionally empowers a few mixed media provisions, for example, constant sound and feature streaming, mixed media conferencing, and intelligent gaming. Broadband associations are likewise being utilized for voice telephony utilizing Voice-Over-Internet Protocol (VOIP) innovation [2]. More praiseworthy broadband access frameworks, for example, fibre-to-the-home (FTTH) and high information rate advanced supporter circle (VDSL), empowers such provisions as diversion quality feature, including highdefinition TV (HDTV) and feature on interest ( VoD).WiMAX is considered to accommodate together fixed and mobile broadband applications. With the introduction of the WiMAX chip in 2003, a wireless network in the local access loop now has the potential to match and on occasion exceed the fixed copper network. WiMAX has the potential of transforming local access capabilities by providing a hotspot that covers a metropolitan area network (MANs); this could be from 20 to $30 \mathrm{~km}$ which is enough coverage, even for the biggest of cities. Also, within the coverage zone a guaranteed Quality of Service (QoS) can be maintained which would allow for such services as IPv4, IpV6, mobile and multimedia communication [3][4].

\section{LITERATURE REVIEW}

In 1998, the Institute of Electrical and Electronics Engineers (IEEE) confined a social occasion called 802.16 to make a standard for Wireless Metropolitan Area Network (WMAN), or remote Metropolitan Area Network [5][6]. The IEEE 802.16 social event took care of a standard that was supported in December 2001. This standard was known as the Wireless MAN-SC and specified a physical layer that utilized single-transporter regulation systems and the layer of Media Access Control with a blast Time Division Multiplexing (TDM) structure that upheld both Time Division Duplexing (TDD) and also the Frequency Division Duplexing (FDD) [7].

Over the years the group continued to expand on the technology and later developed the IEEE 802.16a in 2003. In the wake of finishing this standard, the assembly began deal with stretching out and adjusting it to work in both authorized and permits absolved frequencies in the $2 \mathrm{GHz}$ to $11 \mathrm{GHz}$ extent, which might empower Non-Line of Site (NLOS) organizations [8].

WiMAX is governed by a set of standards which was set by the IEEE, the initial standardization being IEEE 802.16, but currently the newest standards are the IEEE 802.16e 2005, IEEE 802.16d - which provides for fixed and nomadic access, and IEEE 802.16e - which provides for mobility at speeds of up to $120 \mathrm{~km}$ per hour[9][10]. For broadband speeds, WiMAX has a point to multipoint speed of 15 to $70 \mathrm{Mbps}$ which is very good compared to $3 \mathrm{G}$ point to multipoint which provides 10 to $21 \mathrm{kbps}$ or 
Wi-Fi 802.11 point to multipoint which provides 11-54 Besides, this additionally permits preferred QoS taking Mbps.

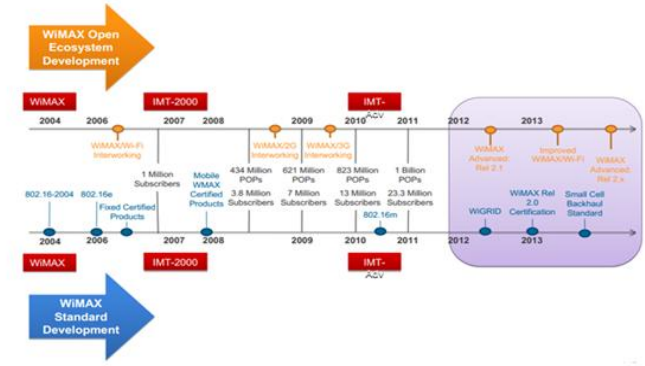

Figure 1: The Evolution of WiMAX Technology and Market Expansion

\section{PROTOCOL LAYER AND ARCHITECTURE}

WiMax has two main topologies namely: Point to Point for backhaul, Point to Multi Point Base station for Subscriber station. In each of these circumstances, Multiple Input Multiple Output (MIMO) receiving wires are utilized. WiMAX gives numerous client requisitions and interfaces like ATM, TDM, Ethernet, VLAN and IP. Moreover, The IEEE 802.16 standard uses the Open System Interconnection (OSI) Network reference model and defines the two lowest layers namely, Physical Layer (PHY) and The Media Access Control (MAC) layer. The MAC layer is sub-divided into three other layers: Convergence sub-layer (CS), the Common Part-Sub layer (CPS) and the Security Sub layer. This standard is capable suit Recurrence Division Duplexing (FDD) or Time Division Multiplexing (TDM) arrangements and likewise takes into account both full and half-duplex terminals. In general, it is found from the 802.16 standard backings three physical layers. The obligatory physical mode is 256-point FFT OFDM (Orthogonal Frequency Division Multiplexing). Alternate modes are Single transporter (SC) and 2048 OFDMA (Orthogonal Frequency Division Multiplexing Access) modes. The relating European standard - the ETSI Hiperman standard characterizes a solitary PHY mode indistinguishable to the 256 OFDM modes in the 802.16d standard [11].

Again, The MAC was produced for a point-to-multipoint remote access environment and can suit conventions such as, IP (Internet Protocol), Ethernet and ATM. The MAC edge structures dynamic uplink and downlink profiles of terminals according to the connection conditions [11]. This is to guarantee an exchange off of limit and constant heartiness. The MAC utilizes a convention information unit of variable length, which expands the principles effectiveness. Numerous MAC convention information unit could be sent as a solitary physical stream to spare over-burden. Additionally, numerous Service information units (SDU) might be sent together to spare on MAC header overhead. By dividing, you can send extensive volumes of information crosswise over edge limits and can ensure a QoS (Quality of Service) of contending administrations. The MAC utilizes a rectifying toward oneself transfer speed solicitation plan to maintain a strategic distance from overhead and acknowledgement delays.

Copyright to IJARCCE care of over the customary recognized plans. The 802.16 MAC protocols perform mostly two assignments: Periodic and A-periodic exercises. Quick exercises (intermittent) like booking, pressing, fracture and ARQ are hard-pressed for time and have hard tight due dates. They must be performed inside a solitary edge.

The moderate exercises, then again, normally execute according to prefixed clocks, yet are not connected with any clocks. They additionally don't have particular time frame or due date.

A. WiMAX Network Architecture

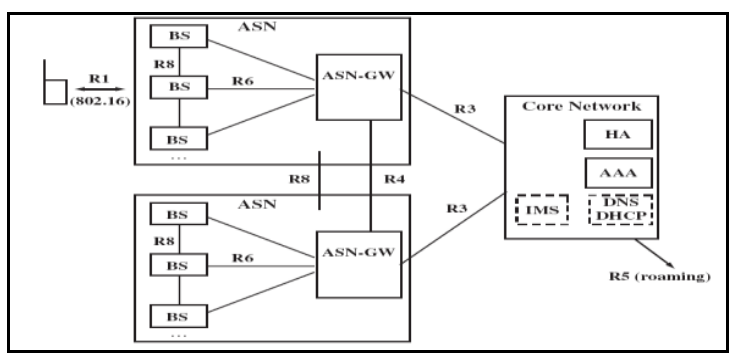

Figure 2: WiMAX Network Architecture

Early WiMAX networks were based on the 802.16d standard which mainly targeted stationary devices with roof-mounted antennas or indoor WiMAX routers with large in-built omni-directional antennas. The air interface standard was enhanced to support subscriber mobility including the handover from one base station to another. This version is referred to as $802.16 \mathrm{e}$ or $802.16-2005$. WiMAX base stations communicate with each other for handover and only require a gateway between the radio network and the core network, the Access Service Network Gateway (ASN-GW) [12]. The ASN-GW is responsible for user management and mobility. As shown in Figure 2 above, the interface between the mobile device and the base station is referred to as the R1 reference point. The radio network base stations are connected to the ASN-GW via the R6 reference point. The R8 reference point allows for smooth handover of connection between base stations.

\section{B. Hardware Systems}

The Hardware systems design from a radio perspective for a WiMAX system adheres to the IEEE 802.16 standard. Some of the hardware antenna technologies used in Fixed and Mobile WiMAX are based on MIMO (Multiple-Input Multiple Output) technology and adaptive antenna technology with beam-forming. In terms of hardware deployment, there will be different methods used for the deployment of Fixed and Mobile WiMAX.

Furthermore, With Fixed WiMAX, the base station is best deployed above the average height of the environment which the signal is been transmitted, this will instigate a Line of Sight or Near Line of sight deployment. It is worth noting at this point that Line of sight propagation will require an area covered by the First Fresnel zone, (an elongated cigar shaped radio beam) this means a coverage area is free from any obstacles, this will be similar to the 
Fixed Wireless Access networks model, in practical application this is seldom the case, usually there is always an obstacle or obstacles in the propagation path between the base station and the customer premises antenna/equipment.

In cases with Non Line of Sight propagation the transmission path to the customer premises equipment has not got a direct path; meaning the customers equipment could be indoors at a level near ground level, or there is a high volume of obstacles in the propagation path. Hardware equipment associated with Non Line of Sight equipment includes Mobile WiMAX terminals, Laptops with PCMCIA Cards and WiMAX enabled mobile phones. With any multiple-user wireless broadband system such as WiMAX, good reception sensitivity and maximized channel capacity for multiple users is a necessary design consideration. The MIMO solution can provide this requirement, the basic evolution of the MIMO system comes out of the SISO (Signal Input Signal Output) system, this system still in common use uses, one data channel, one receiver and one transmitter. The data is sent over a signal data channel with adjustments been made the antenna (Spatial Diversity) for the best signal, this system is an example of a signal carrier technology were the system transmits one digital symbol at a time. Now with the increase throughput demand on wireless broadband, systems are been to cater for multiple digital symbols transmitted at the same time at a low rate per symbol.

Moreover, the modulation scheme OFDM (Orthogonal Frequency Division Multiplexing), together with the MIMO system are the techniques used as a means to provide the technical solution to providing multiple users over a wireless broadband network. The architecture of the MIMO system is based on increasing the amount of information transmitted but using the same bandwidth as if using a signal carrier [13]. An example of this would be if four transmitters are transmitting 4 OFDM independently modulated channels the bandwidth used between transmitter and receiver would reflect this, such as, $4 \mathrm{x}$ bandwidth. In a MIMO transmitted system the four OFDM channels are stacked on top of each other, so the 4 OFDM channels will share the same frequency and therefore you will be able to carry approximately 3.5 times as much data as a single carrier.

In terms of base-station design WiMAX has several advantageous features; one of these features is high data rates; high data rates are achieved by using coding techniques which are advanced, enabling base station to support high data rates over the air, coding techniques used include Orthogonal Frequency Division Multiple Access (OFDMA) and Quadrature Amplitude Modulation QAM. From the base stations point of view the OFDMA method reduces multipath fading, which means the effect of signals arriving at different times from different directions can be dealt with. The base station compared to the subscriber station in a WiMAX system is a complex unit which will support from a couple to thousands of end

users, in the latter case the it is possible for the WiMAX base station to become an integral part of the network providers equipment i.e., working in parallel with wired mainframe network equipment. In fixed WiMAX, a selection of different configurations can apply to base stations depending on what type of environment the base station is working in.

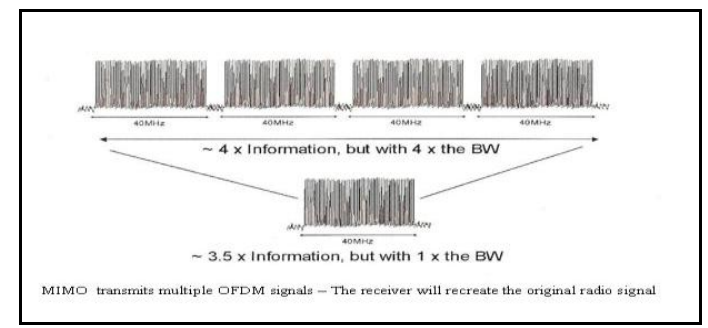

Figure 3: Transmission of multiple OFDM signals using MIMO [13]

Linking deployment environment to end user service we see there are different configurations which may be used. These are as follows - Femto Base Stations, Pico Base Stations, Micro Base stations and Multi-Sector or Macro base stations. Femto Base Stations are base stations that are designed to in the home environment. They will be of a similar size as a home Wi-Fi or wired route, with its back-end connected to the DSL broadband network. The prime coverage target for these kind of base stations is different mobile devices around the home i.e. laptops, PDAs, multimedia streaming. Pico Base Stations are usually placed in an urban city type environment such as airports, shopping centres, office buildings. These units can be mounted on poles and weather proofed if needs be. The radio and baseband equipment will usually be housed in one complete unit, therefore this is a quick deployment cost effective solution. Micro Base Stations are the type of base stations which are usually placed in rural environments or developing countries which have not got a legacy copper telecommunications network, the hardware usually consists of a Rack mounted unit with a radio unit usually mounted on top of a tower. Note, connect between the radio unit and the baseband equipment in the rack is done by a fibre optical cable, the standard for this type of connection is called Open Base Station Architecture Initiative (OBSAI) or Common Public Radio Interface (CPRI). The main reason for this type of connection is that over a long cable length very little signal power is lost.

Multi-sector or Macro base station, as the name implies, these are base stations which have service areas which covers large numbers of end users; each group of end users being defined into different sectors. Macro base station can have a coverage range of 10 to $40 \mathrm{Mbps}$ over a radius of 10 to $15 \mathrm{~km}$. The hardware consists of a Rack mounted unit with the unit having hot swappable cards. The radio unit will be mounted on top of a tower and the connection between rack and radio will be an OBSAI or the CPRI interface. Micro / Multi sector base stations can be thought of to be similar to the $3 \mathrm{G}$ or $4 \mathrm{G}$ base station model Achieving Cost effective. An example of commercial base station design can be seen in below Figure 4. 


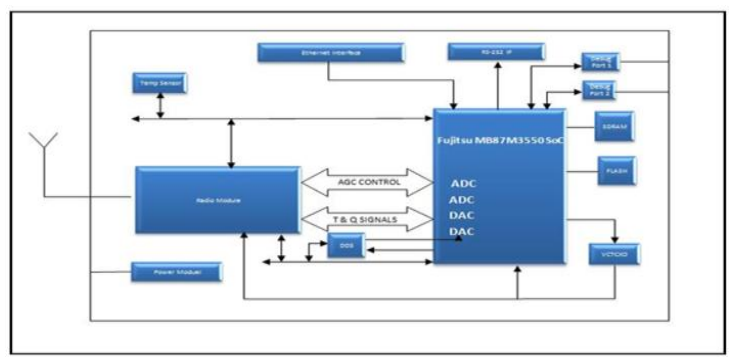

Figure 4: Base Station Design in TDD Mode layout on a SoC chip - Fujitsu WiMAX 802.16-2004 baseband SoC [14]

Moreover, the base station is based on a SoC design (System on a chip), this means that the silicon will contain all the integrated functionality needed to make a base station viable i.e. the chip will have integrated on its silicon sub-straight functional blocks such as described in the next paragraph. The main functional blocks in the system will be the Radio Module, The WiMAX SoC, Ethernet ports, Serial ports, Power module and the Clock circuitry. The ADCs and DACs will have a sampling clock generated by the DDS block and the flash memory block will work in conjunction with the application software and MAC layer functions. The base station can be configured in two modes i.e. TDD - Time Division Duplexing or FDD - Frequency Division Duplexing. In a TDD configured system the uplink and downlink will share the same frequency but can work independent of each other. A TDD frame contains a downlink and uplink sub-frame, sometimes called physical slots which can be defined as the duration of 4 modulation symbols. The TDD system will have the advantage that, on the uplink or downlink the time allocation of the channel can be dynamically adjusted to take into account asymmetrical traffic volumes. In a FDD configured system the uplink and the downlink will be allocated separate frequencies.

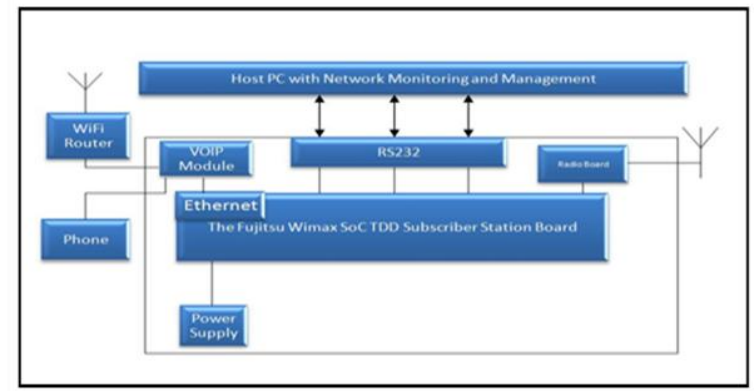

Figure 5: Block diagram of CPE Subscriber unit [14]

An example of commercial used CPE (Customer Premises Equipment) design can be seen in Figure 10 below. The block diagram in Figure 10 is based on the Fujitsu Wimax Subscriber Station Design 802.16-2004 SoC Reference Kit. This is essentially a piece of hardware / firmware which can be used to configure TDD or FDD subscriber stations. An important part of the subscriber station is the MAC level firmware, this is split into two parts i.e. the upper MAC part (UMAC) and the lower MAC part (LMAC). The upper MAC level firmware is responsible for service specific sub-layers and provides privacy, authentication and management services. The lower MAC level firmware will work at the physical set-up and control level, it will help implement things like OFDM drivers and deal with the Physical Service Access Point. A host P.C. or management terminal can talk to the subscriber unit via a serial RS-232 connection or via an Ethernet connection. The application software held on the subscriber station can access data for the subscriber station such as channel selection, Data Rate, Number of Ethernet packets and physical layer frames received or transmitted, Bit Error Rate (BER) and Receiver Sensitivity Signal Strength (RSSI).

\section{APPLICATION AND CHARACTERISTICS OF WIMAX}

\section{A. Data Privacy and Integrity}

Encryption is one of the most important mechanisms to protect the data with confidentiality and integrity. Firstly, this mechanism takes the plaintext from the mobile user such as a text message and then combines this information with a complex algorithm to produce a ciphertext. Accordingly, after that this cipher text is transmitted the data over the wireless network. Now the data which is sent by the customer is fully protected and the reason is that the ciphertext has not able to implicit by an eavesdropper. WiMAX uses AES - Advanced Encryption Standard to protect data through the network. This is an encryption algorithm for securing sensitive information. Figure 6 below shows the procedure for creating cipher text.

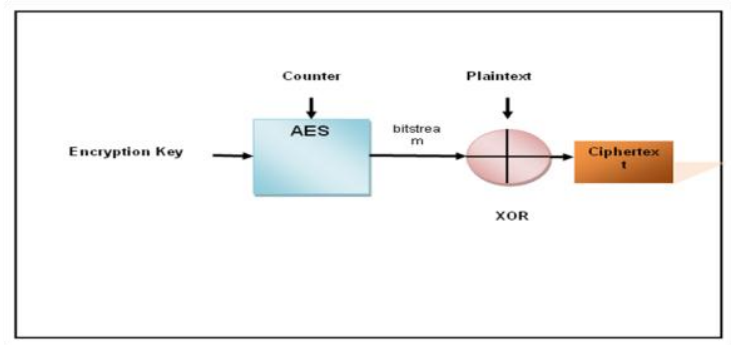

Figure 6: Block Diagram of Advanced Encryption Standard

From Figure 6 above, it is observed that first and foremost an encryption key is obtained for a particular data stream. This encryption key is then combined with a counter to make a bit stream. This bit stream is then used as an input. Likewise, this spot stream is then elite or's with the plaintext to handle the ciphertext. When this process is completed, the data is then transmitted and the recipient of the ciphertext basically reverses the method to recover the original text. However, it is important to note that for this process, the transmitter and receiver must be share the same encryption key.

\section{B. Handoff Mechanism in Mobile WiMAX}

Handoff is one of the most important mechanisms in the Telecommunication field. A host can move from one location to another by the help of handoff technique. This method maintains the subscriber station (SS) switching from one Base Station (BS) to another. This mechanism has been developed, and in general, this technique can be separated into soft handoff and another is hard handoff. Mobile WiMax is used in hard handoff. This technique is illustrated in the figure 7 below: 


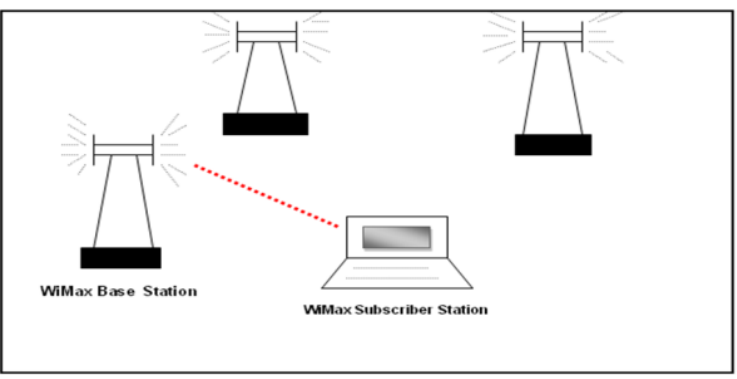

Figure 7: Mobile WiMAX and Hard Handoff

From this figure a connection with a BS is ended before a subscriber station switches to another Base Station and this situation is well-known as a break before make approach. One positive effect on hard handoff is that this mechanism is more bandwidth-efficient than soft handoff; however, it causes quite a long delay. This means that this technique was developed for WiMax to maintain a handoff delay under $50 \mathrm{~ms}$ or more.

C. WiMAX Wireless Service

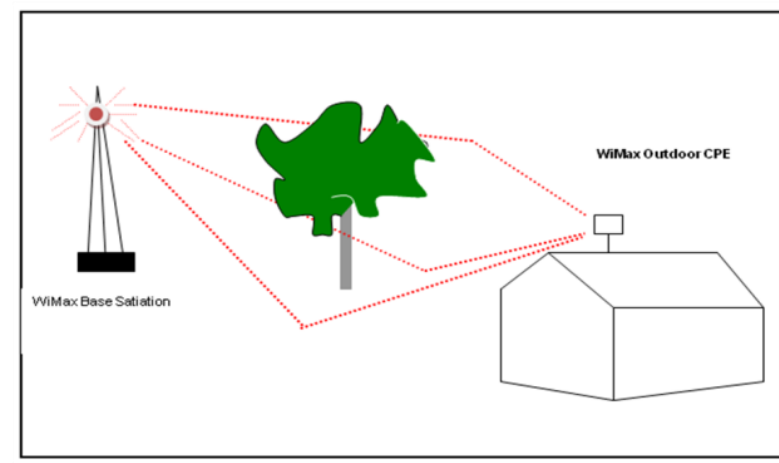

Figure 8: Multipath in NLOS environment

One is the NLOS - non-line-of sight. In this method, a small antenna on the CPE (customer premises equipment) connects to the Wimax tower or BS. For this type of wireless service, WiMax uses a lower frequency range and it is about $2 \mathrm{GHz}$ to $11 \mathrm{GHz}$.

The second method is the LOS means the Line-Of-Sight. In this system a fixed dish antenna is used to point straight at the WiMax tower from a rooftop. This system is more stable and also the connection is stronger. For this reason we can send a lot of data with little or no errors. Here, the transmission use $66 \mathrm{GHz}$ that means this system can support higher frequency.

\section{Authentication}

In WiMAX standard, user devices are referred to as a Customer Premises Equipment (CPE). Whenever a device is powered up, it searches for available networks and get an IP address from the user's home network or from another suitable network.

Before a device is admitted to the network by the ASN$\mathrm{GW}$, an authentication procedure is required; this is illustrated in Figure 9. This is done via a public/private key pair in addition to an X.509 certificate. This key is stored in a safe location on the device itself.

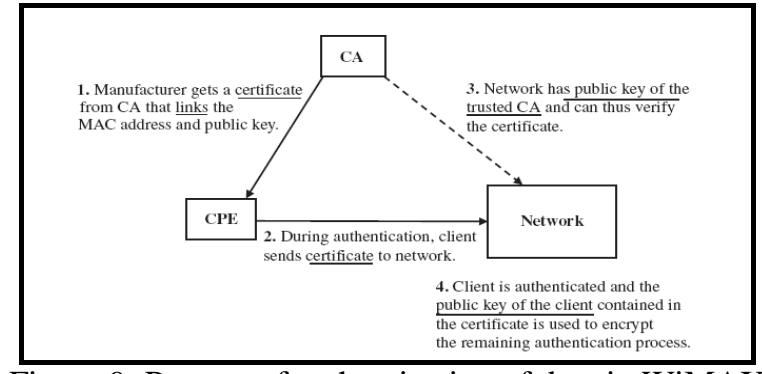

Figure 9: Process of authentication of data in WiMAX networks

\section{RADIO FREQUENCIES DESIGN FOR WIMAX NETWORK}

Proper RF planning and design is essential because in order to investigate the feasibility of deploying a WiMAX Network, one has to be able to assess the number of base stations that will be required in a given geographical area, depending on the services that will be offered, and the number of users that will be utilizing these services. First and foremost, calculation of the link budget, which indicates to what extent the signal might weaken, is discussed. Then, a propagation model is proposed by considering the link budget. The propagation model determines the range and based on this range the required number of base stations can be determined [15].

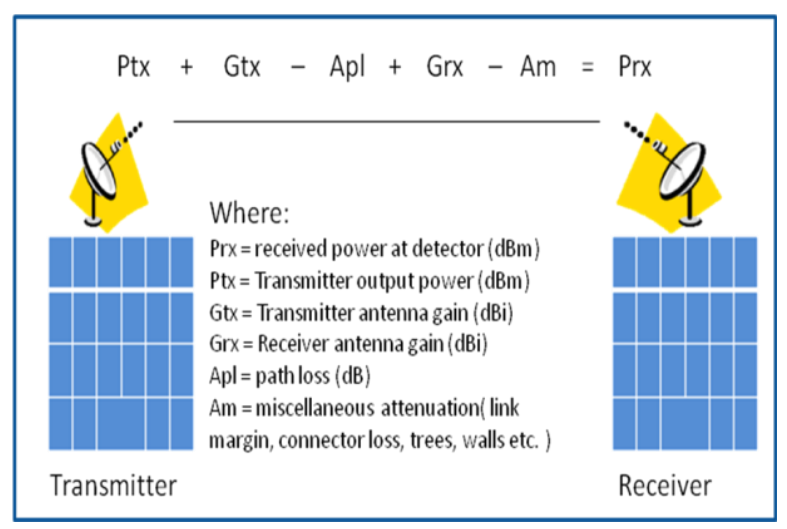

Figure 10: Illustration of Link Budget [16]

\section{A. Link Budget}

A connection budget is the bookkeeping of the greater part of the increases and misfortunes from the transmitter, through the medium (free space, fibre and so on.) to the collector in a telecommunication framework as demonstrated in Figure 11. The connection plan relies on upon a few parameters which are examined beneath.

\section{A link consists of three parts:}

1. Transmitter: the transmitter represents the base station in forward link (FL) communication and represents the CPE in reverse link (RL) communication.

2. Receiver: the receiver is the $\mathrm{CPE}$ in $\mathrm{FL}$ communication and is the BS in RL communication.

3. Media.: The link equation at this stage, neglecting the effect of noise, can be written as:

$\mathrm{P}_{\text {received }}=$ Power of the transmitter + Gain of Transmitting antenna + Gain of the receiving antenna - Sum of all losses. 
The tables below show typical values for these parameters for both the uplink (UL) and downlink (DL) of the Base station (BS) and customer premises equipment (CPE).

Table 1

Transmit and Receive antenna gains for standard 2x2 MIMO Base Stations [17]

\begin{tabular}{|l|l|l|}
\hline & Standard BS & $\begin{array}{l}\text { Base Station with } 2 \times 2 \\
\text { MIMO }\end{array}$ \\
\hline DL Tx Power & $35 \mathrm{dBm}$ & $35 \mathrm{dBm}$ \\
\hline DL Tx antenna gain & $16 \mathrm{dBi}$ & $16 \mathrm{dBi}$ \\
\hline Other DL Tx gain & $0 \mathrm{Db}$ & $9 \mathrm{~dB}$ \\
\hline UL Rx antenna gain & $16 \mathrm{dBi}$ & $16 \mathrm{dBi}$ \\
\hline Other UL Rx gain & $0 \mathrm{~dB}$ & $3 \mathrm{~dB}$ \\
\hline UL receiver noise figure & $5 \mathrm{~dB}$ & $5 \mathrm{~dB}$ \\
\hline
\end{tabular}

Table 2

Transmit and Receive antenna gains for both Portable and Mobile CPE [17]

\begin{tabular}{|l|l|l|}
\hline & Portable CPE & Mobile CPE \\
\hline UL Tx Power & $27 \mathrm{dBm}$ & $27 \mathrm{dBm}$ \\
\hline UL Tx antenna gain & $6 \mathrm{dBi}$ & $2 \mathrm{dBi}$ \\
\hline Other UL Tx gain & $0 \mathrm{~dB}$ & $0 \mathrm{~dB}$ \\
\hline DL Rx antenna gain & $6 \mathrm{dBi}$ & $2 \mathrm{dBi}$ \\
\hline Other DL Rx gain & $0 \mathrm{~dB}$ & $0 \mathrm{~dB}$ \\
\hline DL receiver noise figure & $6 \mathrm{~dB}$ & $6 \mathrm{~dB}$ \\
\hline
\end{tabular}

\section{B. Equivalent Isotropically Radiated Power (EIRP)}

From the parameters for the BS and CPE given in Table 1 and Table 2, the Equivalent isotropically radiated power (EIRP) can then be calculated. The EIRP is characterized as the measure of force that a hypothetical isotropic reception apparatus might transmit to process the crest force thickness watched toward greatest radio wire pick up. EIRP takes into account transmission line and connector losses from the transmitter to the antenna as well as the gain of the antenna [16].

For forward link communication, the BS is the transmitter and its EIRP can be calculated from Table 1 as: EIRP (dB) $=[($ DLT $\times$ Power $)-($ Feeder losses + Connector losses + Jumper losses $)+($ DLT $\times$ Antenna gain + Other DLT $\times$ Gain)] Likewise, for reverse link communication, the CPE is the source for a transmitter and its EIRP be able to be calculated from Table 2.

\section{Receiver Sensitivity}

The receiver sensitivity constitutes an important aspect of a link budget calculation and it is mainly dependent on the following parameters: The thermal noise, the receiver SNR, the Noise Figure and the implementation loss.

Mathematically,

Receiver Sensitivity $S_{R}=$ [Thermal noise $+R_{x} S N R+R_{x}$ noise figure + Implementation loss]

Thermal noise exists in electronic devices due to the random motion of charge carriers in a conductor and is dependent on the bandwidth. It can be estimated as:
Thermal noise $(\mathrm{dBm})=-174+10 \log _{10} \Delta \mathrm{f}$

Where, $\Delta \mathrm{f}$ is act as a bandwidth, in means by hertz, over which the noise is precise, the receiver SNR indicates the signal to noise ratio at the receiver and it generally depends on the modulation scheme. It is worth noting that

WiMAX adaptively selects the modulation system for each and every user and appropriate SNR values are used for different situations. The Noise Figure is a measure of the degradation of the SNR and is a ratio of the actual output noise to the noise that which will remain if the device did not introduce any noise. In short, it represents a key performance indicator of a radio receiver and is given as the implementation loss represents the miscellaneous losses such as: channel estimation loss, phase noise etc and is usually estimated to be around $2 \mathrm{~dB}$.

\section{Margins}

This is characterized as the measure by which an appropriated sign level possibly decreased without creating framework execution to fall underneath a specified threshold value. To determine the link resources, several margins need to be considered such as fade margin, the interference margin and the building penetration loss (BPL) factor. Accordingly, appropriate compensation or allowance is given for these types of impairments to the signal.

\section{E. Link Budget Calculation(Maximum Allowable Path Loss)}

With data obtained from the preceding discussion, the link budget of a WiMAX network can be calculated as follows: For downlink communication, MAPL is specified as:

$\mathrm{MAPL}=\left[\mathrm{EIRP}+\mathrm{CPE}\right.$ DL $\mathrm{R}_{\mathrm{x}}$ antenna gain $+\mathrm{CPE}$ other $\mathrm{DL} \mathrm{R}_{\mathrm{x}}$ gain $-{ }^{\text {Head }} /$ Body loss $-\mathrm{R}_{\mathrm{x}}$ sensitivity - lognormal fading margin - interference margin - Building penetration loss]

For uplink communication, MAPL is specified as:

MAPL $=\left[\right.$ EIRP + BS UL $R_{x}$ antenna gain + BS other UL $R_{x}$ gain $-R_{x}$ Sensitivity - lognormal fading margin - fast fading - interference margin - Building penetration loss + UL sub-channeling gain] A sample link budget for a WiMAX network, showing these salient points is presented in the table below.

Table 4

WiMAX Link Budget Sample [17]

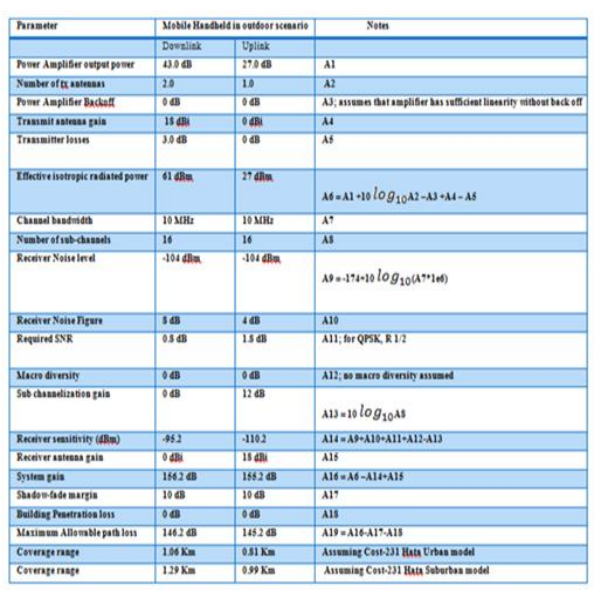


VI. ANALYSIS RESULT AND DISCUSSION Propagation Model:

From Table 3, it can be seen that the Cost-231 Hata propagation model is assumed in deriving the coverage range of our WiMAX network. This model is a modification of the Hata Model and is widely used in cellular networks in the $1,800 / 1,900 \mathrm{MHz}$ range.

This model is applicable for the subsequent range of parameters:

$$
\begin{aligned}
& 150 \mathrm{MHz} \leq \mathrm{f} \leq 2000 \mathrm{MHz} \\
& 30 \mathrm{~m} \leq \mathrm{h}_{\mathrm{b}} \leq 200 \mathrm{~m} \\
& 1 \mathrm{~m} \leq \mathrm{h}_{\mathrm{m}} \leq 10 \mathrm{~m} \\
& 1 \mathrm{Km} \leq \mathrm{d} \leq 20 \mathrm{Km}
\end{aligned}
$$

$\mathrm{f}_{0}$ is the carrier frequency, $\mathrm{h}_{\mathrm{b}}$ is the base antenna height, $\mathrm{h}_{\mathrm{m}}$ is the MS antenna height and $d$ is the distance between the BS and MS.

The median path loss is thus given as: Pl $=\left[46.3+33.9 \log _{10} \mathrm{f}-13.82 \log _{10} \mathrm{~h}_{\mathrm{b}}+(44-\right.$ $\left.\left.6.55 \log _{10} h_{b}\right) \log 10 d-a h_{m}+C_{f}\right]$

The antenna-correction issue for MS is given by:

$\left.\mathrm{A}\left(\mathrm{h}_{\mathrm{m}}\right)=1.11 \log _{10} \mathrm{f}-0.7\right) \mathrm{h}_{\mathrm{m}}-\left(1.56 \log _{10} \mathrm{f}-0.8\right)$

For urban and suburban areas the correction factor $\mathrm{C}_{\mathrm{f}}$ is $3 \mathrm{~dB}$ and $0 \mathrm{~dB}$ respectively. For our system, we assumed a carrier frequency of $2,300 \mathrm{MHz}$, a base station antenna height of $30 \mathrm{~m}$ and a Mobile station height of $1 \mathrm{~m}$. therefore, our coverage range $\mathrm{d}$ was $1.06 \mathrm{Km}$ for downlink given urban propagation environments as indicated in Table 3.

Based on this coverage range, the corresponding cell areas will be: $3 \mathrm{Km}$

Cell area $=3 * \mathrm{~d} 2 * \sin (\pi / 3)$

Therefore, the required number of base stations can be found depending on the geographical size where this system will be deployed and the required data rate that is required for each sector and cell area.

\section{FUTURE DEVELOPMENT OF WIMAX}

One significant chance for Fixed WiMAX in created markets is as an answer for intense T1/E1, partial T1/E1, or higher-pace administrations for the business market. Given that just a little portion of business edifices overall have admittance to fibre, there is a necessity for elective high-transmission capacity answers for venture clients. In the business market, there is interest for symmetrical T1/E1 administrations, the specialized prerequisites of which link and DSL have not met. Altered broadband results utilizing WiMAX could conceivably contend as a part of this business sector and trump landline results regarding time to market, valuing, and element provisioning of transfer speed.

Backhaul for Wi-Fi hotspots: Another intriguing chance for WiMAX in the created world is the possibility to serve as the backhaul association with the expanding Wi-Fi hotspots market. In the United Kingdom and other created markets, a developing number of Wi-Fi hotspots are constantly conveyed openly zones, for example, meeting centres, lodgings, airstrips, and cafes. WiMAX could serve as a quicker and less expensive elective to wired backhaul for these hotspots. Utilizing the point-tomultipoint transmission competencies of WiMAX to serve as backhaul connections to hotspots and could considerably enhance the business case for Wi-Fi hotspots and give further force to hotspot organization. Also, WiMAX could serve as ( $3^{\text {rd }}$ generation) cell backhaul. A potentially large market for fixed broadband WiMAX exists in developing economies such as Brazil, Russia, India, Indonesia, China moreover, several other countries in Asia Latin America, Africa and Eastern Europe.

Consumer and small-business broadband: One of the biggest requisitions of WiMAX within a brief span of time is liable to be broadband access for private, $\mathrm{SOHO}$, and SME markets. Fixed remote offers a few points of interest over universal wired results. These focal points incorporate more level entrance and organization costs; speedier and simpler arrangement and income acknowledgment; capability to manufacture the system as required; easier operational expenses for system support, administration, and operation; and autonomy from the officeholder transporters.

The last venture in the zone system scale is the worldwide region system (GAN). The suggestion for worldwide region system is IEEE 802.20. A correct GAN might work a great deal like today's cell systems, with clients equipped to traverse the nation and still have admittance to the system the entire time. This system might have enough data transmission to offer Internet access tantamount to link modem administration; however it might be approachable to versatile, constantly joined apparatuses like laptops or cutting edge mobile phones.

According to the Intel News Release, they are planning to start making their Centrino laptop processors WiMAX enabled in the next two to three years. If everyone's laptop is WiMAX enabled then it will be less risky for companies to set up WiMAX base stations.

\section{CONCLUSION}

The rapid advancement in the field of communications has led to a parallel rise in demand of communication products and services. This aggregate increase in demand therefore, requires more bandwidth and high-speed solutions. Customers in this information age require access to larger amounts of data, at a faster rate and in any place and time it is required. WiMAX gives this remote result through the meeting of versatile and altered broadband systems through a regular wide zone broadband radio access engineering and adaptable system building design. Likewise, WiMAX gives an extraordinary arrangement of adaptability in system organization choices and administration offerings.

Through the investigation of WiMAX, it has been able to draw up the following conclusions.

WiMAX networks provide higher data rates for both uplink and downlink compared to other wireless solutions 
such as $3 \mathrm{G}$ or $4 \mathrm{G}$ cellular networks. Through the use of MIMO antenna techniques, flexible sub-channelization schemes and advanced coding and modulation, data rates of up to $63 \mathrm{Mbps}$ for uplink and $27 \mathrm{Mbps}$ for downlink can be achieved. The most salient feature of WiMAX is its Quality of Service (QOS). Optimal development of space, frequency and point in time resources ended the air interface on a frame-by-frame basis makes WiMAX a very robust wireless solution. Scalability is also one of the most attractive features that were observed in Wimax Networks. Scalability enables the WiMAX technology to scale to work in different channelization's to comply with various worldwide requirements. The security of WiMAX has also been demonstrated and the future developments explored.

\section{REFERENCES}

[1] Mike Harwood, Comp TIA Network+ + N10-005 Authorized Exam Cram, 4th edi, December 29, 2011, Print ISBN - 978-0-7897-49055

[2] K. Pentikousis, J. Pinola, E. Piri, F. Fitzek, T. Nissilä, and I. Harjula, "Empirical Evaluation of VoIP Aggregation over a Fixed WiMAX Testbed," Proceedings of The 4th International Conference on Testbeds and Research Infrastructures for the Development of Networks \& Communities (TRIDENTCOM), 1820 March, 2008, Innsbruck, Austria

[3] Charles Perkins. IP Mobility Support for IPv4, RFC 3344, 2002

[4] David B. Johnson, Charles Perkins, and Jari Arkko, Mobility Support in IPv6, RFC 3775, 2004

[5] TL Singal. Wireless Communications, ISBN: 9780070681781, MAR-10, Published by the Tata McGrawl Hill Education private Limited, 7 West Patel Nagar, New Delhi 110008

[6] Online available: http://tailieu.vn/doc/fundamentals-of-wimax-p2-244103.html

[7] Dale Stacey. Aeronautical Radio Communication Systems and Networks, 2008, ISBN 978-0-470-01859-0 (HB), John Wiley \& Sons Ltd, The Atrium, Southern Gate, Chichester, West Sussex PO19 8SQ, England

[8] M MALTESH, SWATI GAJAKOSH, WI-MAX TECHNOLOGY ARE YOU READY FOR THE SPEED? ANOTHER MILE STONE IN THE FIELD OF INTERNET ACCESS, 5th International CALIBER -2007, Panjab University, Chandigarh, 0810 February, 2007

[9] Mrs Sukhpreet Kaur, Jasbir Kaur, "Review Paper: To Reduce The Handover Delay In Wimax When The Mobile Station Moves At Higher Speed", International Journal of Computational Engineering Research, Vol, 03, Issue 7, july, 2013

[10] Amitabh kumar.'Mobile Broadcasting with WiMAX: Pricipals, Technology and Applications', Focal Press USA, April 7, 2008, ISBN-10: 0240810406

[11] 2005-2013 Tutorial-Reports.com, [online], available: http://www.tutorial-reports.com/wireless/wimax/protocol.php

[12] Jarno Pinola and Kostas Pentikousis, Mobile WiMAX, The Internet Protocol Journal, Volume 11, No. 2, June 2008

[13] White Paper SISO TO MIMO:Moving Communications from Single-Input Single-Output to Multiple-Input Multiple-Output tmpress.googlecode.com/files/SISO_MIMO_articles.pdf. Retrieved $25^{\text {th }}$ March 2009

[14] [14] Wimax Subscriber Station Design Using the Fujitsu 802.162004 SoC Reference Kit , Fujitsu Microelectronics America, Inc, [online] available: http://www.fujitsu.com/downloads/MICRO/fme/wimax/tech_back_ substation.pdf

[15] [15] B. Sklar, Digital Communications Fundamentals and Applications, 2nd ed., Prentice-Hall, Upper Saddle River, NJ, 2001, pp. 286-290.

[16] R. Freeman, Radio System Design For Telecommunications, $3^{\text {rd }}$ ed., John Wiley, NJ, 2007, pp. 55-58.

[17] Andrews, J., Ghosh A., \& Muhammed, R. (2007), the nuts and bolts of WiMAX. Retrieved March 18, 2009, [online] available: http://www.eetindia.co.in/STATIC/PDF/200708/EEIOL_2007AUG 27_RFD_NETD_EMS_TA_01.pdf?SOURCES=DOWNLOAD

\section{BIOGRAPHIES}

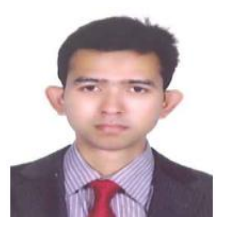

Khan, Mohammad Arifin Rahman, Received his M.Sc in Mobile and Satellite Communications degree from London Metropolitan University, London, United Kingdom, 2010 and has completed the B. Sc (Engg) in Computer Science and Engineering degree from Asian University of Bangladesh, Dhaka, Bangladesh, 2004. As well as, he has made to upgrade his professional career by the specialized certificate CCNA, 2005. His research interests include mobility management, multimedia transmission, and quality-of-service (QoS) provision issues in nextgeneration wireless/mobile networks.

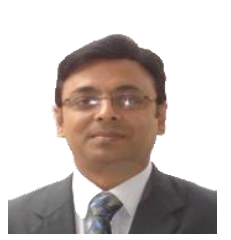

Iqbal, Md. Sadiq, He has completed his M.Sc in Computer Networks degree from Middlesex University, London, Uk, 2005, also has got the another Masters in Computer Science and Engineering degree from National Technical University in Ukraine, Ukraine. Moreover his B.Sc in Computer Science and Engineering has completed from the same university. At the moment he is involved in Center for Excellence in Research, Entrepreneurship \& Teaching at Bangladesh University, Dhaka. 